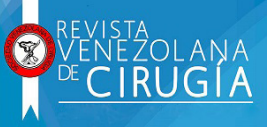

Rev Venez Cir. 74(2): 32-38. 2021

\title{
MODELO INORGÁNICO DE SIMULACIÓN DE GASTROSTOMIAA LAPAROSCÓPICA CON IMPRESIÓN 3D, PARA ADQUIRIR HABILIDADES EN SUTURA INTRACORPÓREA. ESTUDIO CUASI-EXPERIMENTAL
}

\author{
ANDRÉS RODRIGO RUBIN CASTILLO' \\ MIGUEL VASSALLO PALERMO
}

\begin{abstract}
INORGANIC MODEL OF LAPAROSCOPIC GASTROSTOMY SIMULATION WITH 3D PRINTING, TO ACQUIRE SIKILLS IN INTRACORPORAL SUTURE. QUASY EXPERIMENTAL STUDY
\end{abstract}

\section{RESUMEN}

La sutura y el anudado laparoscópico intracorpóreo son las habilidades más difíciles de aprender, las mismas son esenciales para realizar procedimientos laparoscópicos avanzados. Los modelos de entrenamiento laparoscópico permiten facilitar la curva de aprendizaje en un ambiente seguro y sin riesgo para el paciente. La impresión 3D ha revolucionado muchos campos industriales, el presente estudio buscar enlazar la tecnología de impresión 3D con la creación de un modelo anatómico de un estómago inorgánico, para simular una gastrostomía laparoscópica con fines didácticos para los residentes de cirugía general y los cirujanos en formación. Objetivo: Desarrollar un modelo inorgánico de simulación de gastrostomía laparoscópica mediante impresión 3D, como herramienta para la adquisición de habilidades y destrezas en sutura y anudado intracorpóreo. Métodos: Se realizó un estudio de tipo prospectivo, experimental y longitudinal. El autor del presente trabajo, realizó 16 prácticas en caja negra con el modelo de entrenamiento laparoscópico creado y fueron evaluadas mediante la escala GOALS y medición del tiempo de ejecución. Resultados: La puntuación obtenida en la escala GOALS fue de 19 puntos en la primera práctica e incrementó hasta 23 puntos en las últimas prácticas (Tau-C de Kendall $=0,89$ ) siendo estadísticamente significativo ( $p<0,00001)$. El tiempo disminuyó de la primera practica de 18:30 hasta 6:56 minutos en la última (Tau-C de Kendall=-0,49) siendo estadísticamente significativo $(p=0,01)$. Conclusión: El modelo de entrenamiento creado con impresión 3D permite recrear los pasos para realizar una gastrostomía laparoscópica, y demostró ser una herramienta eficaz en la adquisición de habilidades en sutura y anudado intracorpóreo.

Palabras clave: Modelo de entrenamiento, Simulador, Gastrostomía laparoscópica, Impresión 3D, Escala GOALS.

\section{ABSTRACT}

Intracorporeal laparoscopic suturing and knotting are the most difficult skills to learn, and they are essential for performing advanced laparoscopic procedures. Laparoscopic training models facilitate the learning curve in a safe environment without risk for the patient. 3D printing has revolutionized many industrial fields, the present study seeks to link 3D printing technology with the creation of an anatomical model of an inorganic stomach, to simulate a laparoscopic gastrostomy for educational purposes for general surgery residents and surgeons in training. Objetive: develop an inorganic simulation model of laparoscopic gastrostomy using 3D printing, as a tool for the acquisition of skills and abilities in suturing and intracorporeal knotting. Methods A prospective, experimental and longitudinal study was carried out. The author of this work performed 16 black box practices with the laparoscopic training model created and they were evaluated using the GOALS scale and measurement of execution time. Results: The score obtained on the GOALS scale was 19 points in the first practice and increased to 23 points in the last practices (Kendall's Tau-C =0.89). being statistically significant $(p<0.00001)$. The time decreased from the first practice from 18:30 to 6:56 minutes in the last one (Kendall's Tau-C $=-0.49$ ) being statistically significant $(P=0.01)$. Conclusion: The training model created with 3D printing allows to recreate the steps to perform a laparoscopic gastrostomy, and proved to be an effective tool in the acquisition of skills in suturing and intracorporeal knotting.

Key words: Training Model, Simulator, Laparoscopic Gastrostomy, 3D printing, GOALS Scale.
Hospital Universitario de Caracas. Cátedra de Clínica y Terapéutica Quirurgica "B". Especialista en Cirugía General. Correo-e: andresrrc@ hotmail.com. ORCID: 0000-000 1-5755-5996

2. Hospital Universitario de Caracas. Cátedra de Clínica y Terapéutica Quirurgica "B". Especialista en Cirugía General. Profesor Titular de la Universidad Central de Venezuela. ORCID: 0000-000 1-9295-7835
Recepción: 01/08/2021

Aprobación: 23/09/2021

DOI: $10.48104 /$ RVC.2021.74.2.5

muw.revistavenezolanadecirugia.com 


\section{INTRODUCCIÓN}

Los beneficios que ofrece la cirugía laparoscópica respecto a la cirugía convencional son bien conocidos, sin embargo, el cirujano debe superar una larga curva de aprendizaje para alcanzar una serie de habilidades y destrezas quirúrgicas en este campo. (1,2)

Dentro de las habilidades y destrezas que el cirujano debe adquirir, la sutura y el anudado laparoscópico intracorpóreo son esenciales para poder realizar procedimientos laparoscópicos avanzados. Sin embargo, el desarrollo de estas habilidades suele ser difícil por las limitaciones propias de la cirugía mínimamente invasiva. (3)

La cirugía mínimamente invasiva mediante laparoscopia presenta desafíos para el operador, enfrentando obstáculos como: pérdida de percepción de la profundidad, disminución del rango de movimiento, retroalimentación háptica limitada, visión en dos dimensiones y en ocasiones necesidad de usar posiciones poco ergonómicas. $(4,5)$

Sumado a estas limitantes, la sutura y anudado intracorpóreo laparoscópico en particular son habilidades con una curva de aprendizaje empinada, y es difícil adquirirlas dentro de los programas de formación actuales de cirugía general. (6)

Esto se debe principalmente a que los alumnos tienen poca familiarización con procedimientos laparoscópicos avanzados que requieren sutura intracorpórea y al hecho de que la práctica de esta habilidad avanzada dentro del quirófano consume mucho tiempo $(7,8)$. En la actualidad existe una importante brecha entre el nivel de práctica y las destrezas mínimas con las que se debería egresar de los programas de especialización en cirugía general, respecto a la realidad; esto es un punto importante para promover el uso de modelos de entrenamiento quirúrgicos. $(9,10)$

Adicionalmente, la mayor razón para la incorporación de la simulación en el entrenamiento quirúrgico es el imperativo ético de dar a los pacientes la mejor atención. Aunque se entiende que los residentes y cirujanos en formación eventualmente desarrollarán habilidades técnicas al tratar a los pacientes, estos no deben estar sujetos a la posibilidad de daño cuando otros métodos de capacitación están disponibles para la adquisición de habilidades. La simulación asegura que se haya practicado antes de que los aprendices traten a pacientes reales. (11-13)

Así lo expresa Nagendran et al. en su metaanálisis realizado en la Escuela Universitaria de Londres. Donde compararon el entrenamiento con simulador versus ningún entrenamiento. Afirmando que, el entrenamiento con simulador disminuye el tiempo requerido para realizar una tarea laparoscópica, aumenta la exactitud, reduce los errores, y mejora el rendimiento general. (14)

De igual forma, Toledo et al. ratifican que el entrenamiento en simulación laparoscópica conlleva una mejoría significativa en términos de habilidad técnica, desarrollo procedimental y tiempo en todos Ios participantes, independientemente de su nivel formativo o experiencia previa, así lo demuestran en su trabajo. (15)

Van Sickle et al., evaluaron el impacto de un plan de estudios mediante simulación en sutura y anudado laparoscópico. Los resultados de este estudio sugieren que el tiempo, en lugar de los errores o manipulaciones de la aguja, fue la mejor medida de la habilidad de sutura y anudado laparoscópico. (16)

En nuestro país, también se han llevado a cabo estudios en este campo y así lo describe Castro, quien realizó un modelo de entrenamiento laparoscópico con materiales inertes, afirmando que demostró la posibilidad de adquirir destrezas y habilidades para la sutura y el anudado laparoscópico intracorpóreo. (17)

La impresión 3D ha demostrado su utilidad en la creación de modelos de entrenamiento laparoscópico, así lo refiere Casas et al, quienes realizaron diferentes modelos anatómicos impresos en 3D del conducto cístico y sus variantes, planteando que sus modelos son una solución de bajo costo para un simulador de entrenamiento en cirugía laparoscópica. (18)

Igualmente, Chen et al., sostienen que el diseño digital y la impresión 3D para la reconstrucción anatómica durante la planificación quirúrgica, conduce a una comprensión anatómica óptima y facilita la simulación quirúrgica, concluyendo que esta herramienta puede influir potencialmente en los resultados positivos para los pacientes. (19)

\section{Impresión 3D}

La impresión 3D consta de un grupo de tecnologías de fabricación por adición donde un objeto tridimensional es creado a partir de un diseño digital, mediante la superposición de capas sucesivas de material. Las impresoras 3D son por lo general más rápidas, más baratas y más fáciles de usar que otras tecnologías de fabricación por adición. El material de fabricación se deposita en planos virtuales realizados por diseño asistido por ordenador, que la máquina utiliza sucesivamente como guía para la impresión. (20)

Existe un gran número de tecnologías disponibles para la impresión 3D que están en competencia entre ellas. Sus principales diferencias se encuentran en la forma en la que las diferentes capas se unen para crear piezas. Generalmente las consideraciones principales son velocidad, costo del prototipo impreso, costo de la impresora 3D, elección del material, asi como la capacidad o no para elegir el color. De las diferentes tecnologías disponibles para realizar impresión 3D, el modelado por deposición fundida, es el que está teniendo mejor acogida en el sector empresarial y doméstico. (20)

Modelado por deposición fundida: Usa termoplásticos en filamentos previamente extruidos, mediante una tobera deposita el material fundido sobre una estructura soporte generalmente en sentido horizontal, capa a capa. Cuando se acaba una capa, se empieza la siguiente. No es posible una vez que se ha impreso una capa, descender en altura para hacer una capa inferior (20).

Desde 2003 ha habido un gran crecimiento en la venta de impresoras 3D. Esta tecnología ha revolucionado diversos campos industriales. El campo de la medicina con sus ramas quirúrgicas, incluyendo a la cirugía general, no son ajenas a este progreso. En este contexto, el Instituto de Investigación en Atención de Emergencias en el 2015 situó a la impresión 3D en el segundo 
puesto de las 10 tecnologías de mayor impacto sanitario (22), y la Administración de Alimentos y Medicamentos de Estados Unidos ha publicado recomendaciones para su aplicación clínica. (23)

\section{Sistema de evaluación}

La escala de Evaluación Global de Habilidades Laparoscópicas (GOALS) fue desarrollada por Vassiliou et al, en la Universidad McGill en Montreal y evalúa la ejecución de un procedimiento en cirugía mínimamente invasiva en cinco ítems (percepción de profundidad, destreza bimanual, eficiencia, manejo de tejidos, y autonomía). Consiste en una escala de Likert de 1 a 5 puntos, incluyendo una descripción en los puntajes 1, 3 y 5 para cada ítem de la intervención laparoscópica, donde "1" representa el nivel más bajo de rendimiento, y" 5 " se considera un rendimiento ideal. La puntuación total de la escala es la suma de las puntuaciones de cada uno de los 5 ítems, para una puntuación máxima total de 25 puntos el cual equivale a un excelente desempeño. (Tabla 1). (24, 25)

1abla 1. Escala de Evaluación Global de Habilidades Laparoscópicas (GOALS)

- Percepción de profundidad
(1 Punto) Constantemente sobrepasa el objetivo, oscilaciones
amplias, lento para corregir.
( 3 Puntos) Algunas veces sobrepasa o pierde el objetivo, sin
embargo, rápido para corregir.
(5 Puntos) Dirige exactamente los instrumentos hacia el objetivo.
- Destreza bimanual
(1 Punto) Usa sólo una mano, ignora la mano no dominante,
pobre coordinación entre ambas manos.
(3 Puntos) Usa ambas manos, pero no optimiza la interacción
entre ambas manos.
(5 Puntos) Utiliza expertamente ambas manos de una manera
complementaria para proveer una exposición óptima.
- Eficiencia
(1 Punto) Esfuerzos inciertos e ineficaces, muchos movimientos
provisionales, constantemente cambia de foco o persiste sin progreso.
(3 Puntos) Lento, pero los movimientos planeados son
azonablemente organizados.
(5 Puntos) Seguro y eficiente, mantiene el foco en la tarea hasta
que esta es realizada.

\section{Manejo del tejido}

(1 Punto) Movimientos ásperos, tejido rasgado, injuria structuras adyacentes, control pobre del instrumento de prehensión deslizamiento frecuente del instrumento de prehensión.

(3 Puntos) Manejo de los tejidos razonablemente bien, mínimo rauma a tejidos adyacentes (es decir, sangría ocasional o deslizamiento del instrumento de prehensión).

(5 Puntos) Buen manejo del tejido, aplica la tracción apropiada herida insignificante a estructuras adyacentes.

\section{Autonomía}

(1 Punto) Incapaz de completar la tarea entera, inclusive hast on guía verbal.

(3 Puntos) Capaz de completarla tarea sin peligro con guí moderada

(5 Puntos) Capaz de completar la tarea independientement in faltas

Fuente: Navarro, S.

\section{Objetivo general}

Crear un modelo inorgánico de simulación de gastrostomía laparoscópica mediante impresión 3D, como herramienta para generar la adquisición de habilidades y destrezas quirúrgicas de sutura y anudado intracorpóreo.

\section{Objetivos especificos}

1. Reproducir los pasos necesarios para hacer una gastrostomía laparoscópica, en sesiones prácticas de caja negra realizadas por el autor.

2. Analizar el puntaje obtenido según la escala GOALS en cada sesión práctica realizada

3. Medir el tiempo en que se completa cada sesión práctica realizada.

4. Evaluar el desempeño durante las sesiones prácticas con el modelo de entrenamiento, comparando el puntaje obtenido según la escala GOALS y el tiempo en que se realizó cada práctica.

\section{Aspectos éticos}

El presente estudio usó un modelo inorgánico y se llevó a cabo en un laboratorio de entrenamiento laparoscópico. Por ende, no se está sujeto a los principios de la declaración de Helsinki.

\section{MÉTODOS}

Estudio de tipo prospectivo, experimental y longitudinal.

\section{Población y muestra}

Se realizaron 16 prácticas en caja negra en el Laboratorio de Entrenamiento de Cirugía Laparoscópica de la Cátedra de Clínica y Terapéutica “B”, del Hospital Universitario de Caracas, y la evaluación fue realizada por un cirujano experto. Las sesiones prácticas fueron realizadas por el autor del trabajo.

\section{Procedimientos}

Se contrató a la empresa: Soluciones VePrint3D C.A., para realizar el diseñó y posterior impresión 3D del modelo anatómico de un estómago, bajo la directriz del autor.

El proceso de diseño digital lo realizó el ingeniero aeronáutico Kelvin Soler (Figura 1) y el proceso de impresión 3D, el ingeniero aeronáutico Andreas Díaz, mediante modelado por deposición fundida (Figura 2).

Inicialmente se planteó el uso de un termoplástico flexible denominado poliuretano, para elaborar el modelo de estómago en su totalidad, sin embargo, luego de las pruebas funcionales se optó por construir el estómago en dos piezas (cara anterior y posterior) completamente con material rígido (ácido poliláctico) y que en la cara anterior del cuerpo gástrico contara con una ventana que permitiera colocar una membrana de látex (zona en donde se realizó el ejercicio de sutura laparoscópica), la membrana de látex se confeccionó recortando una bolsa respiratoria 


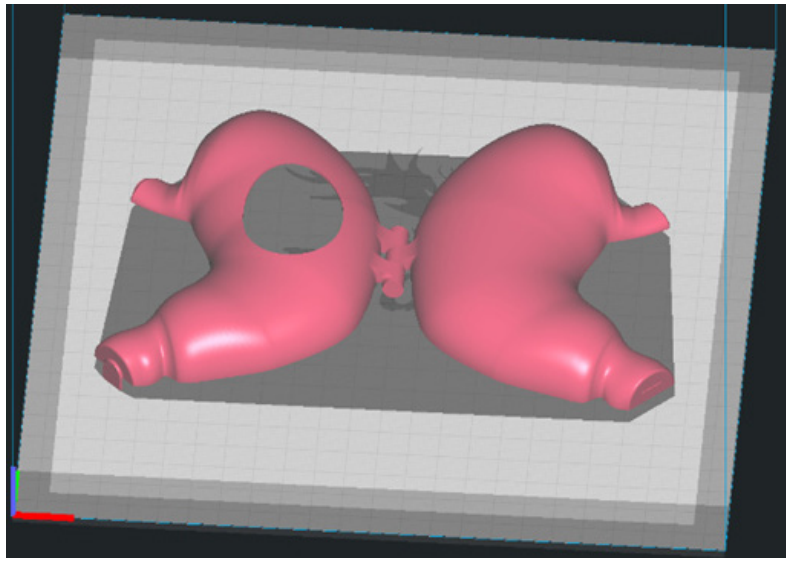

Figura 1. Diseno digital definitivo del estómago (Fuente propia)

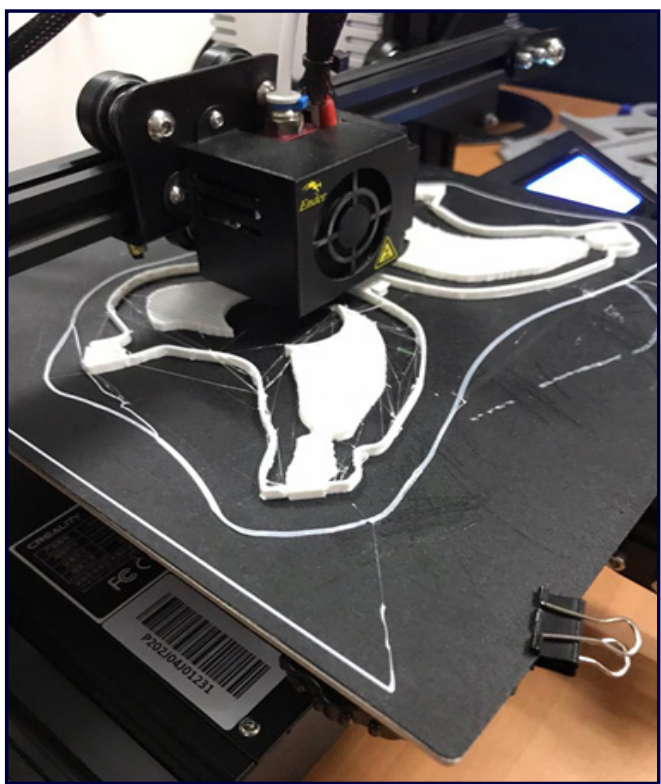

Figura 2. Proceso de impresion 3D del estomago (Fuente propia)

desechable y fue adherida al estómago con silicón caliente Adicionalmente se incorporó una bisagra que permitió articular las cara anterior y posterior del estómago, facilitando el recambio de la membrana de látex después de cada práctica. (Figura 3)

Posteriormente, se recreó una gastrostomía laparoscópica usando como principio la técnica de Stamm, sin embargo, por fines prácticos se modificó la misma y se realizó una sola sutura en bolsa de tabaco, y no se realizó fijación a la pared abdominal.

Cada práctica que se realizó en la caja negra, constó del siguiente procedimiento: primero se fijó el estómago al piso de la caja negra (Figura 4), luego en su pared anterior se realizó una sutura en bolsa de tabaco con sutura de seda 3-0, a continuación en el medio de la sutura en bolsa de tabaco se hizo una incisión con tijera laparoscópica. Posteriormente, a través de la incisión hecha se introdujo una sonda de Foley número 14, se llenó el

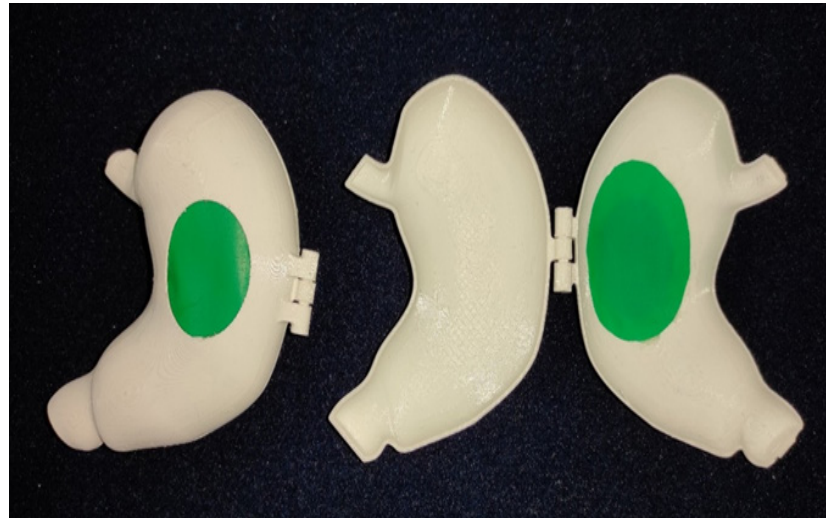

Figura 3. Modelo definitivo del estómago fabricado con impresión 3D con membrana de látex incorporada(Fuente propia)

balón de la misma con 5 cc de aire, después se realizó 3 nudos para cerrar la sutura y asegurar la sonda, luego se efectuó una suave tracción sobre la sonda de Foley para verificar que se encontrara bien fijada al estómago, y el último paso fue cortar los cabos de la sutura con tijera laparoscópica. (Figura 5).

\section{Tratamiento estadistico adecuado}

A partir de una base de datos en el programa Microsoft Excel, se realizaron gráficos de dispersión, con línea de tendencia en cuanto a la direccionalidad de las variables ordinales a asociar con el programa estadístico SPSS 26 (SPSS Inc., USA) se aplicó la prueba estadística no paramétrica Tau-C de Kendall, considerando como estadísticamente significativo cuando $\mathrm{p}<0,05$.

\section{RESULTADOS}

La puntuación en la escala GOALS obtenida en la primera práctica fue de 19 puntos y luego fue aumentando progresivamente, en la tercera práctica fue de 21 puntos y posterior a la novena práctica su puntuación incremento a 23 puntos y se mantuvo así hasta finalizar la totalidad de las prácticas.

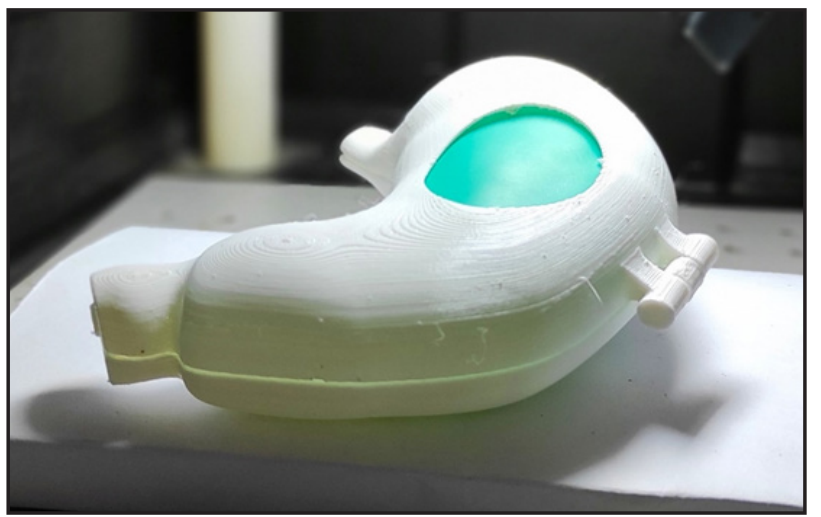

Figura 4. Estómago impreso en 3D dentro de la caja negra laparoscópica, fijado al piso de la misma (Fuente propia) 


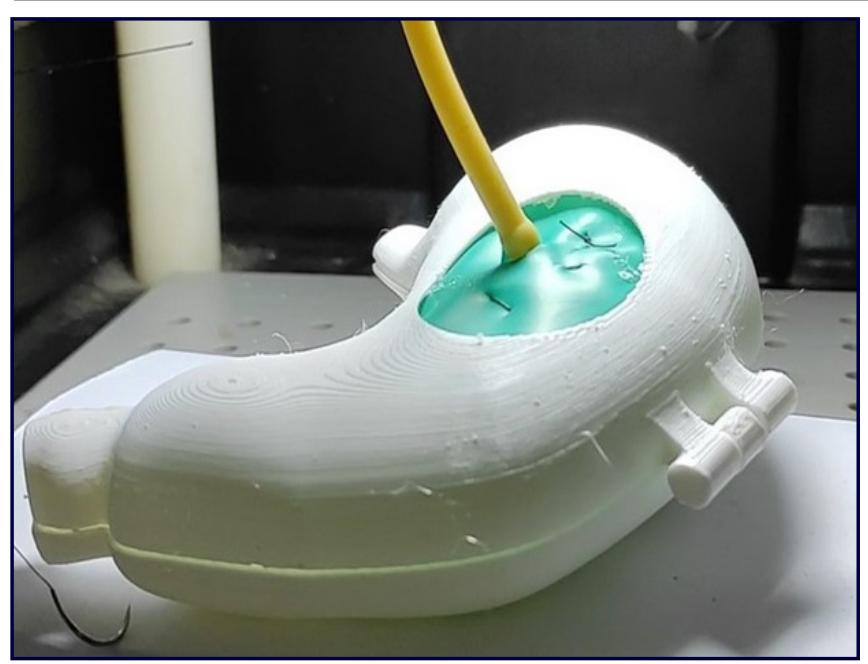

Figura 5. Vista del procedimiento de gastrostomia finalizado. (Fuente propia)

La asociación entre el número de prácticas realizadas y el puntaje obtenido fue directamente proporcional (Tau-C de Kendall =0,89), y estadísticamente significativa ( $p<0,00001$ ) (Tabla 2)

\section{Tabla 2. Puntuación total de la escala GOALS de acuerdo al número de prácticas}

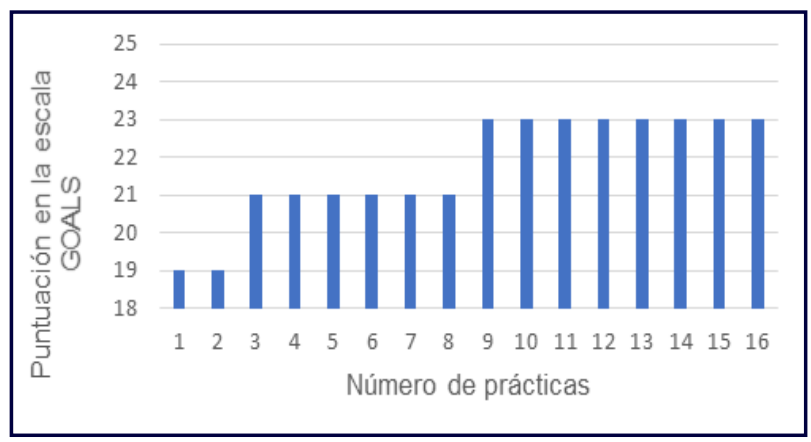

Tau-C de Kendal = 0,89; valor de $\mathrm{p}<0,00001$ (significativo)

En relación al tiempo quirúrgico se observó que a mayor número de prácticas menor tiempo de ejecución, la primera práctica se realizó en un tiempo de 18:30 minutos y la última se realizó en 6:56 minutos. Demostrando una asociación inversamente proporcional entre tiempo y número de prácticas (Tau-C de Kendall = -0,49) siendo estadísticamente significativa ( $p=0,01$ ) (Tabla 3).

Al comparar el tiempo quirúrgico con la puntuación de la escala GOALS, durante el transcurso de las prácticas realizadas, se encontró una relación inversamente proporcional entre el tiempo y puntuación GOALS, evidenciando que a medida que progresaron el número de prácticas realizadas, disminuyó el tiempo quirúrgico y aumento el puntaje obtenido, lo cual fue estadísticamente significativo ( $p<0,0001$ ) (Tabla 4)

\section{DISCUSIÓN}

Las indicaciones para las intervenciones mediante cirugía laparoscópica han seguido en expansión en los últimos años, sin embargo, su curva de aprendizaje más empinada hace necesario el adiestramiento y entrenamiento constantes en esta técnica, tanto por cirujanos en formación como por cirujanos laparoscópicos expertos. $(7,8)$

La utilización de modelos de entrenamiento laparoscópicos mediante simulación, plantea una solución a lo antes mencionado e incrementaría las habilidades laparoscópicas del cirujano en cuestión. $(11,12)$

El procedimiento de gastrostomía laparoscópica no es uno de los procedimientos realizados con mayor frecuencia en cirugía general, sin embargo, se escogió para esta investigación ya que es fácil y simple de recrear, y su simulación permite realizar el ejercicio de sutura y anudado intracorpóreo. Al realizar los entrenamientos en caja negra con el modelo creado, se observó que su ejecución permite reproducir con gran fidelidad los pasos necesarios para realizar una gastrostomía laparoscópica, el tamaño resultó adecuado al espacio de la caja negra y la ventana de látex donde se realizó la sutura, presentó una textura y consistencia bastante realista permitiendo el paso de la aguja de forma cómoda.

Diversos estudios han manifestado que posterior a realizar prácticas en modelos de entrenamiento laparoscópico y una vez que han sido dominados los pasos de un procedimiento ex vivo, el cirujano progresaría favorablemente en su curva de aprendizaje, adquiriendo las destrezas y habilidades necesarias que le permitirían mejorar su desempeño dentro de quirófano, esto se conoce como "transferencia de entrenamiento". (15, 27, 30)

Los resultados fueron estadísticamente significativos y de acuerdo a los mismos se hace evidente una notable mejoría del tiempo quirúrgico, incluso solo después de realizar las primeras seis prácticas y un importante desarrollo de las habilidades y destrezas laparoscópicas.

Resulta interesante comparar estos resultados con los obtenidos en otros trabajos nacionales en donde se llevaron a cabo entrenamientos con diferentes modelos de simulación,

Tabla 3. Tiempo quirurgico medido en minutos de acuerdo al número de prácticas.

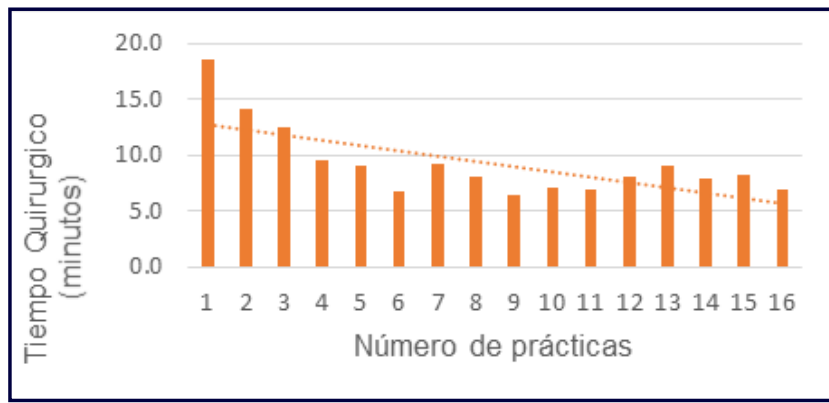

Tau- $C$ de Kendal =-0,49; valor de $\mathrm{p}=0,01$ (significativo) 
Tabla 4. Asociación entre tiempo quirurgico y la

puntuación total en la escala GOALS de acuerdo al número de prácticas

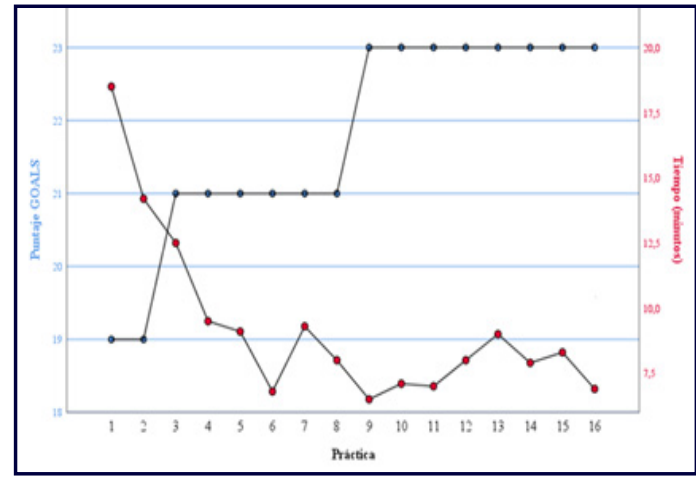

Valor de $\mathrm{p}=0,0001$ (significativo).

como: apendicetomía laparoscópica por abordaje de una sola incisión (28), cura laparoscópica de hernia inguinal (29), instrumentación laparoscópica de la vía biliar. (30) Todos mostraron una notable mejoría del tiempo quirúrgico y mejoría del desempeño de las destrezas laparoscópicas después de realizar los entrenamientos. Esto se encuentra en concordancia con los resultados de este trabajo.

De igual forma, los resultados obtenidos, se correlacionan con los diversos estudios internacionales donde ampliamente se ha demostrado que las prácticas con modelos de entrenamiento inanimados, acortan la curva de aprendizaje, reducen los errores y mejoran el tiempo quirúrgico. (14-16)

Haber superado los desafíos que se presentaron y los resultados obtenidos constituyen un estímulo para seguir por esta línea de investigación, y a partir de ello seguir innovando con nuevos modelos de entrenamiento creados con impresión 3D, que pudieran contribuir en el adiestramiento laparoscópico.

\section{CONCLUSIÓN}

Se logró crear un modelo de un estómago inorgánico mediante impresión 3D, para simular una gastrostomía laparoscópica. Y el modelo creado permitió reproducir con gran realismo y fidelidad los pasos quirúrgicos de este procedimiento.

Demostró ser una herramienta muy útily valiosa, disminuyendo el tiempo quirúrgico de realización del procedimiento y aumentando significativamente las habilidades laparoscópicas.

La tecnología de impresión 3D como herramienta para fabricar modelos anatómicos de enseñanza, probó ser un método eficaz para la creación de un modelo simulación de gastrostomía laparoscópica, fácilmente reproducible, práctico y de bajo costo.

Los residentes de cirugía general y cirujanos en formación se podrían beneficiar al utilizar este modelo de entrenamiento como una herramienta complementaria de aprendizaje en cirugía laparoscópica.

Se podría estudiar la aplicación del modelo de entrenamiento creado con impresión 3D para gastrostomía laparoscópica, en una población de cirujanos en formación, en nuestro hospital o incluso de otros centros del país.

La adquisición de habilidades laparoscópicas básicas no es un requisito previo para el entrenamiento en sutura intracorpórea, por lo que recomiendan que es beneficioso incluir el entrenamiento en sutura intracorpórea desde el inicio del programa de formación quirúrgica.

Se deberá continuar en la búsqueda de modelos que permitan desarrollar al máximo las capacidades y habilidades del cirujano, en particular a cada uno de los procedimientos utilizados en la práctica de la cirugía.

\section{Conflicto de Intereses}

Los autores declaran no tener conflicto de interés.

\section{REFERENCIAS}

1. Spaner S, Warnock G. A brief history of endoscopy, laparoscopy, and laparoscopic surgery. Laparoendosc Adv Surg Tech A [Internet] 1997 [citado 9junio 2020]; 7(6):369-373. Disponible en: https://doi org/10.1089/lap. 1997.7.369

2. Himal H. Minimally invasive (laparoscopic) surgery. Surg Endosc [Internet]. 2002 [citado 5junio 2020]; 16(12):1647-1652. Disponible en: https://doi.org/10.1007/s00464-00 1-8275-7

3. Lucena J, Coronel P. Formación del cirujano en técnicas quirúrgicas laparoscópicas. RFM [Internet]. 2006 [citado 11 junio 2020]; 29(2):97-102. Disponible en: http://ve.scielo.org/scielo. php?script=sci_arttext\&pid=\$0798-0469200600020000 1 \&lng=es

4. Stefanidis D, Hope W, Korndorffer J, Markley S, Scott D. Initial Laparoscopic Basic Skills Training Shortens the Learning Curve of Laparoscopic Suturing and Is Cost-Effective. J Am Coll Surg [Internet] 2010 [citado 21 junio 2020]; 210(4):436-440. Disponible en: https:// doi.org/10.1016/j.jamcollsurg.2009.12.015

5. Dehabadi M, Fernando B, Berlingieri P. The use of simulation in the acquisition of laparoscopic suturing skills. Int J Surg [Internet]. 2014 [citado 23 mayo 2020]; 12(4):258-268. Disponible en: https://doi. org/10.1016/j.jisu.2014.01.022

6. Harold K, Harold B, Backus C, Pratt B, Heniford B. Prospective randomized evaluation of surgical resident proficiency with laparoscopic suturing after course instruction. Surg Endosc [Internet] 2002 [citado 4 julio 2020]; 16(12): 1729-1731. Disponible en: https:// doi.org/10.1007/s00464-002-8832-8

7. Bridges $M$, Diamond $D$. The financial impact of teaching surgical residents in the operating room. Am J Surg [Internet]. 1999 [citado 16 junio 2020]; 177(1):28-32. Disponible en: https://doi.org/10.1016/ s0002-9610(98)00289-x

8. Babineau T, Becker J, Gibbons G, Sentovich S, Hess D, Robertson S. The "cost" of operative training for surgical residents. JAMA [Internet] 2004 [citado 31 mayo 2020]; 139(4):369-370. Disponible en: https:// doi.org/10.100 1/archsurg.139.4.366

9. Blencowe N, Parsons B, Hollowood A. Effects of changing work patterns on general surgical training over the last decade. Postgrad Med J [Internet]. 2011 [citado 13 junio 2020]; 87(1034):795-799. Disponible en: https://doi.org/10.1136/postgradmedj-20 1 1-130297 10. Bell R, Biester T, Tabuenca A, Rhodes R, Cofer J, Britt L. Operative 
experience of residents in US general surgery programs: a gap between expectation and experience. Ann Surg [Internet]. 2009 [citado 19 mayo 2020]; 249(5):719-724. Disponible en: https://doi. org/10.1097/SLA.0b013e3181a38e59

11. Ziv A, Wolpe P, Small D, Glick S. Simulation-based medical education: an ethical imperative. Academic medicine: journal of the Association of American Medical Colleges [Internet]. 2003 [citado 8 junio 2020]; 78(8):783-788. Disponible en: https://doi. org/10.1097/00001888-200308000-00006

12. Issenberg S, McGaghie W, Hart I, Mayer J, Felner J, Petrusa E, et al. Simulation technology for health care professional skills training and assessment. JAMA [Internet]. 1999 [citado 22 junio 2020]; 282(9):861-866. Disponible en: https://doi.org/10.1001/ jama.282.9.861

13. de Montbrun S, Macrae H. Simulation in Surgical Education. Clin Colon Rectal Surg [Internet]. 2012 [citado 13 junio 2020]; 25(3):156165. Disponible en: https://doi.org/10.1055/s-0032-1322553

14. Nagendran M, Gurusamy K, Toon C, Davidson B. Laparoscopic surgical box model training for surgical trainees with limited prior laparoscopic experience. Cochrane Database Syst Rev [Internet]. 2014 [citado 6 julio 2020]; (3):CD0 10478. Disponible en: https:// doi.org/10.1002/14651858.CD0 10478.pub2

15. Toledo E, Martín J, Magadán C, López A, Fernández R, Regaño $S$, et al. Influencia de la experiencia previa en los beneficios del entrenamiento quirúrgico laparoscópico basado en la simulación. Cir Esp [Internet]. 2019 [citado 24 junio 2020]; 97(6):314-319. Disponible en: https://doi.org/10.1016/j.ciresp.2018.12.004

16. Van Sickle K, Baghai M, Huang I, Goldenberg A, Smith D, Ritter E. Construct validity of an objective assessment method for laparoscopic intracorporeal suturing and knot tying. Am J Surg [Internet]. 2008 [citado 6 junio 2020]; 196(1):74-80. Disponible en: https://doi. org/10.1016/j.amjsurg.2007.08.058

17. Castro D. Sutura y anudado laparoscópico intracorpóreo: Impacto del uso de un modelo de entrenamiento en la adquisición de habilidades. Repositorio Institucional de la Universidad Central de Venezuela [Internet]. 2014 [citado 9 junio 2020]; Disponible en: http://saber.ucv.ve/handle/123456789/7906

18. Casas C, Zuñiga A, López R, Sánchez A, Gogeascoechea A, Muñoz G, et al. 3D-printed anatomical models of the cystic duct and its variants, a low-cost solution for an in-house built simulator for laparoscopic surgery training. Surg Radiol Anat [Internet]. 2021 [citado 18 enero 2021]. Disponible en: https://doi.org/10.1007/s00276-020-02631-3

19. Chen S, Ong C, Malguria N, Vricella L, Garcia J, Hibino N. Digital Design and 3D Printing of Aortic Arch Reconstruction in HLHS for Surgical Simulation and Training. World J Pediatr Congenit Heart Surg [Internet]. 2018 [citado 18 enero 2021]; 9(4):454-458. Disponible en: https://doi.org/10.1177/2150135118771323

20. Impresión 3D [Sitio Web]. Wikipedia, la enciclopedia libre, 2021 [citado 12 febrero 2021]. Disponible en: https://es.wikipedia.org/ wiki/Impresión_3D

21. Mantrana G, Jacobo O, Hartwing D, Giachero V. Modelos de impresión tridimensional en la planificación preoperatoria y en la enseñanza académica de las fracturas mandibulares. Cir plást iberolatinoam [Internet]. 2018 [citado 1 junio 2020]; 44(2):193-201. Disponible en: http://dx.dqoi.org/10.4321/ s0376-78922018000200010.

22. Top 10 healthcare technology issues for 2015. [Sitio Web] Emergency Care Research Institute. 2015 [citado 13 junio 2016]. Disponible en: https://wmw.ecri.org/press/Pages/Google-Glass-3D-printing-ECRI-Institute- Top-10-Healthcare-Technology-20 1 5.aspx

23. Technical considerations for additive manufactured devices [Sitio Web]. U.S. Department of Health and Human Services. Food and Drug Administration. 2016 [citado 13 junio 2020]. Disponible en: http://mww.fda.gov/downloads/MedicalDevices/ DeviceRegulationandGuidanc e/GuidanceDocuments/UCM499809. pdf

24. Navarro S, Gabrielli N, Varas C. Evaluación Objetiva de las Habilidades Técnicas en Cirugía. ARS Med [Internet]. 2018 [citado 18 junio 2020]; 43(3):6-14. Disponible en: https://doi.org/10.1 1565/arsmed. v43i3.1112

25. Vassiliou M, Feldman L, Andrew C, Bergman S, Leffondré K, Stanbridge D, et al. A global assessment tool for evaluation of intraoperative laparoscopic skills. Am J Surg [Internet]. 2005 [citado 26 junio 2020]; 190(1):107-1 13. Disponible en: https://doi. org/10.1016/j.amjsurg.2005.04.004

26. Castro D. Sutura y anudado laparoscópico intracorpóreo: Impacto del uso de un modelo de entrenamiento en la adquisición de habilidades. Repositorio Institucional de la Universidad Central de Venezuela [Internet]. 2014 [citado 9 junio 2020]; Disponible en: http://saber.ucv.ve/handle/123456789/7906

27. Korndorffer J Jr, Dunne J, Sierra R, Stefanidis D, Touchard C, Scott D. Simulator training for laparoscopic suturing using performance goals translates to the operating room. J Am Coll Surg [Internet]. 2005 [citado 25 febrero 2021]; 201 (1):23-9. Disponible en: https:// doi.org/0.1016/j.jamcollsurg.2005.02.021

28. Millán P, Visconti M. Modelo de entrenamiento para apendicectomía laparoscópica mediante abordaje de una solo incisión con uso de instrumentos articulados flexibles. Repositorio Institucional de la Universidad Central de Venezuela [Internet]. 2014 [citado 9 junio 2020]; Disponible en: http://saber.ucv.ve/handle/123456789/7867

29. Luque, E. Cura laparoscópica de hernia inguinal: validación de un modelo de entrenamiento. Repositorio institucional de la Universidad Central de Venezuela. [Internet]. 2016 [citado 10 junio 2020]; Disponible en: http://saber.ucv.ve/handle/123456789/15121

30. Sánchez A, Benítez P, Rodríguez O, Pujadas Z, Valero R. Desarrollo de un modelo de entrenamiento para la instrumentación laparoscópica de la vía biliar guiada por fluoroscopia. Rev Venez Cir 2006; 59:66-71. 